\title{
Megakaryocytes Increased in Bone Marrow
}

National Cancer Institute

\section{Source}

National Cancer Institute. Megakaryocytes Increased in Bone Marrow. NCI Thesaurus.

Code C35168.

A laboratory test result indicating abnormally high numbers of megakaryocytes in the bone marrow. 